\title{
Recorded Penicillin Allergy and Risk of Mortality: a Population-Based Matched Cohort Study
}

\author{
Kimberly G. Blumenthal, MD, MSc $c^{1,2,3,4}$, Na Lu, MPH ${ }^{1,5}$, Yuqing Zhang, DSc ${ }^{1,3,4,5}$, \\ Rochelle P. Walensky, MD, MPH ${ }^{2,3,4,6}$, and Hyon K. Choi, MD, DrPH
}

'Division of Rheumatology, Allergy, and Immunology, Department of Medicine, Massachusetts General Hospital, Boston, MA, USA; ${ }^{2}$ Medical Practice Evaluation Center, Massachusetts General Hospital, Boston, MA, USA; ${ }^{3}$ Harvard Medical School, Boston, MA, USA; ${ }^{4}$ The Mongan Institute, Massachusetts General Hospital, Boston, MA, USA; ${ }^{5}$ Clinical Epidemiology Research and Training, Boston University School of Medicine, Boston, MA, USA; 'Division of Infectious Diseases, Department of Medicine, Massachusetts General Hospital, Boston, MA, USA.

\section{Abbreviations \\ GP}

HR

J Gen Intern Med 34(9):1685-7

DOI: $10.1007 / \mathrm{s} 11606-019-04991-y$

(c) Society of General Internal Medicine 2019

\section{INTRODUCTION}

Approximately $10 \%$ of patients have a recorded penicillin allergy, a label that results in prescribing shifts towards less effective and broader-spectrum antibiotics. Patients with a recorded penicillin allergy have an increased risk of drugresistant organisms, healthcare-associated infections, and antibiotic treatment failures. ${ }^{1-3}$ When evaluated, more than $95 \%$ of patients with a recorded penicillin allergy are not allergic. ${ }^{4}$ We examined the relation between a newly recorded penicillin allergy and the risk of death.

\section{METHODS}

We performed a population-based matched cohort study among adult participants enrolled in the Healthcare Improvement Network (January 1, 1995-December 31, 2015), an electronic database of 11.1 million patients registered with general practitioners (GPs). ${ }^{5}$ We identified patients with their first GP-recorded allergy to a penicillin antibiotic linked to a prior penicillin prescription, and selected up to five age $( \pm$ 1 year)-, sex-, and dataset entry time ( \pm 1 year)-matched penicillin users without a recorded penicillin allergy. ${ }^{2}$ Recorded penicillin allergy could be reported by patients, family members, or other providers to GPs, or could represent a GP-diagnosed penicillin allergy. For cases, the index date was the date of first penicillin allergy entry; for comparators, the matched index date was within 1 year of penicillin prescription. The primary outcome was all-cause mortality. Potential confounding variables including demographics, lifestyle factors, medications, allergies, comorbidities, and healthcare utilization were assessed at baseline. Follow-up

Published online April 22, 2019 time was calculated from the index date to the date of death or the end of the study, whichever occurred first.

We used Cox proportional hazard models to calculate hazard ratios (HRs) for the relation of penicillin allergy status to the risk of all-cause mortality. The multivariable models adjusted for all confounders assessed at baseline; the Charlson Comorbidity Index was used to adjust for major medical comorbidities. Analyses were performed using SAS, version 9.4 (SAS Institute, Cary, NC).

\section{RESULTS}

We identified 63,690 patients with a recorded penicillin allergy and 237,167 comparators (Table 1). Patients with a recorded penicillin allergy were generally similar to comparators, except for other antibiotic allergies, which were more common in patients with a recorded penicillin allergy.

During 6 years of mean follow-up time, 8773 patients with a recorded penicillin allergy and 28,793 patients without a recorded penicillin allergy died (Table 2). The age-, sex-, and entry time-matched HR for all-cause mortality for patients with a recorded penicillin allergy was 1.18 (1.15 to 1.21$)$ compared to those without recorded penicillin allergy. The matched and multivariable adjusted HR for all-cause mortality for patients with a recorded penicillin allergy was 1.14 (1.12 to 1.17) compared with those without recorded penicillin allergy.

\section{DISCUSSION}

A recorded penicillin allergy was associated with a $14 \%$ increased risk of death. When allergy tested, $95 \%$ of adults with a recorded penicillin allergy are not allergic, an incongruence that exists because penicillin intolerances or viral exanthems may be recorded as allergies, and beta-lactam allergy wanes over time. ${ }^{4,} 6$ Some of the harms associated with a recorded penicillin allergy may be modifiable with improved antibiotic prescribing in patients with penicillin allergy labels. 
Table 1 Cohort Characteristics According to Penicillin Allergy Status

\begin{tabular}{|c|c|c|}
\hline Characteristics* & $\begin{array}{l}\text { Recorded } \\
\text { penicillin allergy } \\
(n=63,690)\end{array}$ & $\begin{array}{l}\text { No recorded } \\
\text { penicillin allergy } \\
(n=237,167)\end{array}$ \\
\hline Age (years), mean (SD) & $54.8(20.1)$ & $55.0(20.0)$ \\
\hline Female & $42,995(67.5)$ & $164,661(69.4)$ \\
\hline \multicolumn{3}{|l|}{ Body mass index } \\
\hline$<18.5$ & $1453(2.3)$ & $5040(2.1)$ \\
\hline $18.5-24.9$ & $21,669(34.0)$ & $82,567(34.8)$ \\
\hline $25.0-29.9$ & $17,806(28.0)$ & $66,395(28.0)$ \\
\hline$>30.0$ & $12,272(19.3)$ & $42,952(18.1)$ \\
\hline Unknown & $10,490(16.5)$ & $40,213(17.0)$ \\
\hline \multicolumn{3}{|c|}{ Socioeconomic Deprivation Index score } \\
\hline 1 & $15,904(25.3)$ & $59,048(25.3)$ \\
\hline 2 & $13,548(21.6)$ & 50,419 (21.6) \\
\hline 3 & $12,789(20.4)$ & $46,915(20.1)$ \\
\hline 4 & 11,042 (17.6) & 40,504 (17.4) \\
\hline 5 & $7218(11.5)$ & $27,268(11.7)$ \\
\hline \multicolumn{3}{|l|}{ Smoking status } \\
\hline None & $33,036(51.9)$ & $124,242(52.4)$ \\
\hline Past & $9762(15.3)$ & $35,860(15.1)$ \\
\hline Current & $17,233(27.1)$ & $61,235(25.8)$ \\
\hline \multirow{2}{*}{\multicolumn{3}{|c|}{ Alcohol use }} \\
\hline & & \\
\hline None & $12,197(19.2)$ & $43,362(18.3)$ \\
\hline Past & $811(1.3)$ & $2599(1.1)$ \\
\hline Current & $39,038(61.3)$ & $145,961(61.5)$ \\
\hline Unknown & $11,644(18.3)$ & $45,245(19.1)$ \\
\hline \multicolumn{3}{|l|}{ Comorbidities } \\
\hline Diabetes & $5482(8.6)$ & $19,580(8.3)$ \\
\hline Renal disease & 4197 (6.6) & $14,628(6.2)$ \\
\hline Hemodialysis & $68(0.1)$ & $199(0.1)$ \\
\hline Malignancy & $3564(5.6)$ & $12,304(5.2)$ \\
\hline Liver disease & $1100(1.7)$ & $3334(1.4)$ \\
\hline Charlson Comorbidity & $0.5(1.1)$ & $0.5(1.0)$ \\
\hline Index, mean $(\mathrm{SD})$ & & \\
\hline \multicolumn{3}{|l|}{ Medications } \\
\hline $\begin{array}{l}\text { Number of antibiotic } \\
\text { prescriptions (1 year), } \\
\text { mean (SD) }\end{array}$ & $4.0(4.8)$ & $3.5(4.1)$ \\
\hline $\begin{array}{l}\text { Number of antibiotic } \\
\text { prescriptions ( } 2 \text { years), } \\
\text { mean (SD) }\end{array}$ & $4.4(5.3)$ & $3.8(4.5)$ \\
\hline Systemic corticosteroid & $21,140(33.2)$ & $76,865(32.4)$ \\
\hline \multicolumn{3}{|l|}{ Other drug allergies } \\
\hline Cephalosporins & $781(1.2)$ & $1190(0.5)$ \\
\hline Other antibiotic allergies & $5711(9.0)$ & $10,731(4.5)$ \\
\hline Nursing home living & $80(0.1)$ & $428(0.2)$ \\
\hline $\begin{array}{l}\text { General practitioner visits, } \\
\text { mean (SD) }\end{array}$ & $5.3(4.4)$ & $4.7(4.0)$ \\
\hline $\begin{array}{l}\text { Hospitalizations, mean } \\
\text { (SD) }\end{array}$ & $0.8(2.0)$ & $0.6(1.7)$ \\
\hline
\end{tabular}

*Data displayed as $n$ (\%) unless otherwise specified

${ }^{\dagger}$ Matched on age, sex, and entry time; matched index date within 1 year of penicillin prescription

${ }^{*}$ Townsend Deprivation Index, grouped into quintiles from 1 (least deprived) to 5 (most deprived)

$S D$, standard deviation

Limitations of this analysis include the potential for residual confounders. While we adjusted for Charlson Comorbidity Index, antibiotic prescriptions, and healthcare utilization at baseline, it remains conceivable that penicillin allergy may still be a marker for increased contact with the healthcare system. Although we identified that a recorded penicillin allergy was associated with an increased mortality, we were unable to examine cause-specific mortality. Nevertheless, allcause mortality is itself important because it represents the overall net health impact of a recorded penicillin allergy.
Table 2 Association of a Recorded Penicillin Allergy on All-Cause Mortality

\begin{tabular}{lll}
\hline \hline & \multicolumn{2}{l}{ Recorded penicillin allergy } \\
\cline { 2 - 3 } & Yes & No \\
\hline Number of patients & 63,690 & 237,167 \\
Follow-up time in years (mean \pm SD) & $6.0 \pm 4.28$ & $6.1 \pm 4.26$ \\
Number of person-years & 379,369 & $1,441,303$ \\
All-cause mortality & & \\
Number of deaths & 8773 & 28,793 \\
Age-, sex-, and entry time-matched & $1.18(1.15$ to & 1.0 \\
HR (95\% CI) & $1.21)$ & (reference) \\
Multivariable adjusted HR (95\% & $1.14(1.12$ to & 1.0 \\
CI)* & $1.17)$ & (reference) \\
\hline
\end{tabular}

*Age-, sex-, and entry time-matched and adjusted for age, sex, body mass index, socioeconomic status, smoking alcohol, Charlson Comorbidity Index, hemodialysis, antibiotic prescriptions, corticosteroid use, other antibiotic allergies, nursing home living, general practitioner visits, and hospitalizations

$S D$, standard deviation; HR, hazard ratio; $C I$, confidence interval

A $14 \%$ increase in death overall was identified for patients with a recorded penicillin allergy. Once a penicillin allergy is recorded, patients receive less effective and/or more toxic antibiotics for subsequent infections. ${ }^{1-3}$ Clinical outcomes for the over 30 million Americans with a recorded penicillin allergy may well improve if penicillin allergy verification programs could eliminate these labels from the majority of people who carry them.

Corresponding Author: Kimberly G. Blumenthal, MD, MSc; The Mongan Institute, Massachusetts General Hospital, Boston, MA, USA (e-mail:kblumenthal@mgh.harvard.edu).

Funding Information This work was supported by $\mathrm{NIH}$ K01AI125631, the American Academy of Allergy Asthma and Immunology Foundation, and the MGH Claflin Distinguished Scholars Award. Dr. Walensky was supported by the Steven and Deborah Gorlin MGH Research Scholars Award.

\section{Compliance with Ethical Standards:}

Conflict of Interest: Dr. Blumenthal reports a licensed clinical decision support tool for beta-lactam allergy evaluation that is used institutionally at Partners HealthCare System and licensed by Persistent Systems. All remaining authors declare that they do not have a conflict of interest.

Disclaimer: The content is solely the responsibility of the authors and does not necessarily represent the official views of the National Institutes of Health, the American Academy of Allergy Asthma and Immunology Foundation, nor the MGH Executive Committee on Research.

\section{REFERENCES}

1. MacFadden DR, LaDelfa A, Leen $\mathbf{J}$, et al. Impact of reported beta-lactam allergy on inpatient outcomes: a multicenter prospective cohort study. Clin Infect Dis. 2016;63(7):904-910.

2. Blumenthal KG, Lu N, Zhang Y, Li Y, Walensky RP, Choi HK. Risk of meticillin resistant Staphylococcus aureus and Clostridium difficile in patients with a documented penicillin allergy: population based matched cohort study. BMJ. 2018;361:k2400.

3. Jeffres MN, Narayanan PP, Shuster JE, Schramm GE. Consequences of avoiding beta-lactams in patients with beta-lactam allergies. J Allergy Clin Immunol Pract. 2016;137(4):1148-1153. 
4. Sacco KA, Bates A, Brigham TJ, Imam JS, Burton MC. Clinica outcomes following inpatient penicillin allergy testing: A systematic review and meta-analysis. Allergy. 2017;72(9):1288-1296.

5. Jick SS, Kaye JA, Vasilakis-Scaramozza C, et al. Validity of the general practice research database. Pharmacotherapy. 2003;23(5):686-689.
6. Trubiano JA, Adkinson NF, Phillips EJ. Penicillin allergy is not necessarily forever. JAMA. 2017;318(1):82-83.

Publisher's Note Springer Nature remains neutral with regard to jurisdictional claims in published maps and institutional affiliations. 